\title{
INEXTENSIBLE DOMAINS
}

\author{
YOAV KALLUS
}

\begin{abstract}
We develop a theory of planar, origin-symmetric, convex domains that are inextensible with respect to lattice covering, that is, domains such that augmenting them in any way allows fewer domains to cover the same area. We show that originsymmetric inextensible domains are exactly the origin-symmetric convex domains with a circle of outer billiard triangles. We address a conjecture by Genin and Tabachnikov about convex domains, not necessarily symmetric, with a circle of outer billiard triangles, and show that it follows immediately from a result of Sas.
\end{abstract}

In a series of papers from 1946 to 1947, Kurt Mahler developed a theory of planar, origin-symmetric, star-like domains that are irreducible with respect to lattice packing, that is, domains such that taking away any piece allows more domains to be packed in the same area [5, 6]. In particular, he was interested in the problem of identifying convex domains such that every convex domain with lower area may be packed at a greater number per unit area than the domain in question. This is the subject of Reinhardt's conjecture [7]. In particular, Mahler showed that the disk is not such a domain, even if the domains of lower area to which we compare it are restricted to a small neighborhood of the disk with respect to Hausdorff distance [6].

Inspired by the work of Mahler, we develop a theory of planar, originsymmetric, convex domains that are inextensible with respect to lattice covering, that is, domains such that adding any piece allows fewer domains to cover the same area. We find that the inextensible domains are simply those with a circle of outer billiard triangles, a family of domains studied previously by Genin and Tabachnikov [3]. The analogue of Reinhardt's domain for covering is simply the ellipse: any originsymmetric convex domain can cover the plane with no larger number per unit area than an ellipse of the same area, as can be easily shown using a classical result of Sas [8, 9]. In other words, the ellipse covers the plane with the least efficiency.

Genin and Tabachnikov conjecture that out of convex domains, not necessarily symmetric, with a circle of critical triangles of a fixed area,

Date: June 5, 2018. 


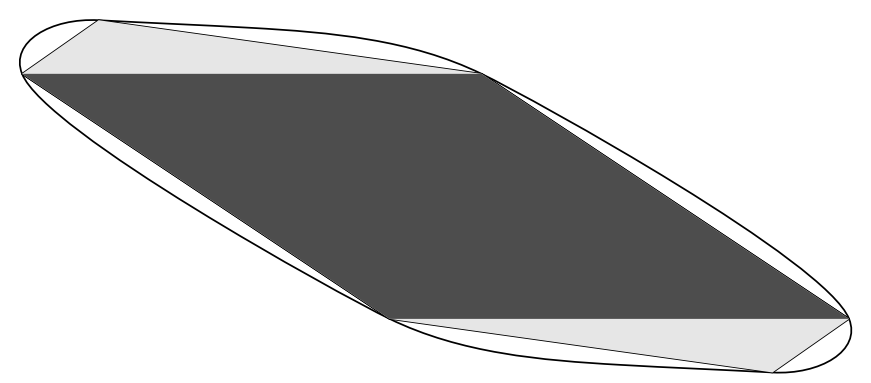

Figure 1. The parallelogram $K$ (dark gray) and a proper superdomain $K^{\prime}$ (white). Any such superdomain includes a hexagonal (or degeneratly, parallelogrammatic) superdomain (light gray), so the parallelogram is inextensible.

the ellipse provides the upper bound for the area of the domain [3]. This also follows easily from the result of Sas.

We call $K$ a symmetric convex domain (below just "domain"), if $K$ is a convex compact subset of $\mathbb{R}^{2}$ such that $K=-K$. A lattice $\Lambda=B \mathbb{Z}^{2}$ is the image of the integer lattice $\mathbb{Z}^{2}$ under a nonsingular linear map $B$. The determinant $d(\Lambda)$ of a lattice $\Lambda=B \mathbb{Z}^{2}$ is given by $|\operatorname{det}(B)|$ and is independent of the basis $B$ used. We will use the Hausdorff metric as a distance between domains: $\delta\left(K, K^{\prime}\right)=\min \{\varepsilon$ : $\left.K \subseteq K^{\prime}+\varepsilon D, K^{\prime} \subseteq K+\varepsilon D\right\}$, where $D$ is the unit disk. As the distance between lattices we use the distance between the closest two bases: $\delta\left(\Lambda, \Lambda^{\prime}\right)=\min \left\{\left\|B-B^{\prime}\right\|: \Lambda=B \mathbb{Z}^{2}, \Lambda=B^{\prime} \mathbb{Z}^{2}\right\}$, where $\|\cdot\|$ is the Hilbert-Schmidt norm.

The lattice $\Lambda$ is called $K$-covering if $K+\Lambda=\mathbb{R}^{2}$. Of all $K$-covering lattices there is at least one lattice $\Lambda_{c}$ such that $d(\Lambda) \leq d\left(\Lambda_{c}\right)$ whenever $\Lambda$ is $K$-covering [4. We call such a lattice a critical lattice for $K$ and its determinant is called the critical determinant of $K$ and denoted $\Delta(K)$. Since every point of the unit cell of the lattice is covered at least singly, $\Delta(K) \leq \operatorname{area}(K)$, with equality if $K$ tiles the plane. Whenever $K^{\prime} \supseteq$ $K$, any critical lattice of $K$ is also $K^{\prime}$-covering, and therefore $\Delta\left(K^{\prime}\right) \geq$ $\Delta(K)$. We say that $K$ is extensible if there is a domain $K^{\prime}$ containing $K$ but different from it that has the same critical determinant as $K$. Otherwise, we say $K$ is inextensible.

Let us begin with an example of an inextensible domain: a parallelogram (see Figure 1). Since the parallelogram tiles the plane, the critical determinant of a parallelogram is equal to its area. Let $K$ be a parallelogram and let $K^{\prime}$ be a proper superdomain. By definition there must be a point $\mathbf{x} \in K^{\prime} \backslash K$. Let $K^{\prime \prime}=\operatorname{conv}(K, \pm \mathbf{x})$. Since $K^{\prime \prime}$ is 


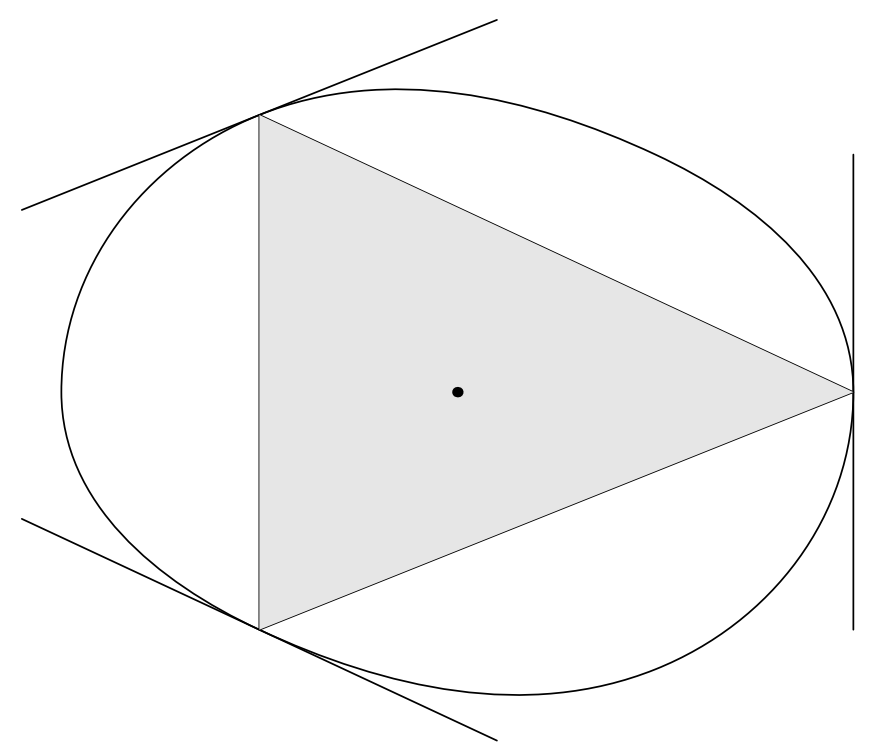

Figure 2. A convex, centrally symmetric domain, with one its critical triangles (light gray). The triangle must contain the origin in its interior (unless the domain is a parallelogram) and there must be support lines through each of the three vertices that are parallel to the opposite sides of the triangle.

either a parallelogram of a hexagon, its critical determinant is equal to its area and therefore $\Delta(K)<\Delta\left(K^{\prime \prime}\right) \leq \Delta\left(K^{\prime}\right)$.

Bambah and Rogers have showed that if $T$ is a triangle inscribed in $K$, maximizing the area among all triangles inscribed in $K$, then the critical determinant of a domain $K$ is equal to twice the area of $T$ [1]. We call such a triangle a critical triangle of $K$ (see Figure 2). Consequently, any lattice which is critical for the (possibly degenerate) hexagon $\operatorname{conv}(T,-T)$ is critical for $K$. Any critical triangle $T$ must include the origin, otherwise a reflection of one of its vertices through the opposite side yields a triangle of equal area but with one vertex in the interior of $K$. When $K$ is a parallelogram, $T$ may have the origin on its perimeter. When $K$ is not a parallelogram, the origin must be in the interior of $T$, since otherwise the hexagon $\operatorname{conv}(T,-T)$ degenerates to a parallelogram which is simultaneously a proper subdomain of $K$ and of equal critical determinant, contradicting the inextensibility of parallelograms. Similarly, the hexagon $\operatorname{conv}(T,-T)$ must also not degenerate to a parallelogram by having one of the vertices of $T$ lie on 


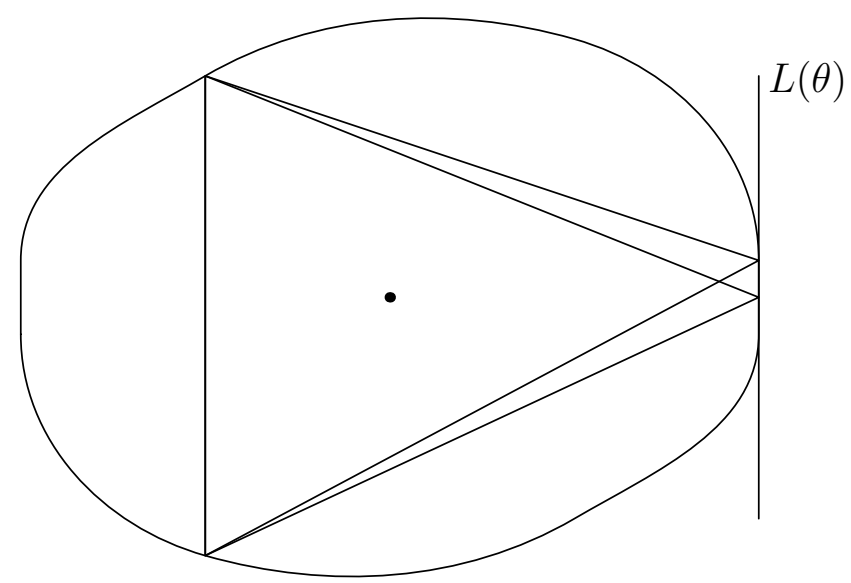

Figure 3. Two triangles anchored at $L(\theta)$ : inscribed triangles with one vertex on $L(\theta)$ and one side parallel to $L(\theta)$, maximizing the area among all such triangles. As shown in Lemma 1, the base of all triangles anchored at a given support line is the same. Additionally, if the support line intersects $K$ at a point, then there is a unique triangle anchored at $L(\theta)$.

a side of $-T$ and therefore the relative interiors of all sides of $T$ must be interior to $K$.

Let $\mathbf{u}_{\theta}=(\cos \theta, \sin \theta)$ be a point on the unit circle at angle $\theta$ from the $x$-axis. The support height of a domain $K$ in the direction $\mathbf{u}_{\theta}$ is given by $h(\theta)=\max _{\mathbf{x} \in K}\left\langle\mathbf{x}, \mathbf{u}_{\theta}\right\rangle$. The support line $L(\theta)$ is the line $\left\{\mathbf{x} \in \mathbb{R}^{2}:\left\langle\mathbf{x}, \mathbf{u}_{\theta}\right\rangle=h(\theta)\right\}$, and it intersects the boundary of $K$ but not its interior. In order for the critical triangle to have maximal area among inscribed triangles, there must be a support line through each of its vertices that is parallel to opposite side (see Figure 2).

Lemma 1. Let $L(\theta)$ be a support line of $K$. Consider all triangles $\mathbf{x y z}$ inscribed in $K$ in such a way that $\mathbf{x} \in L(\theta)$, and the side $\mathbf{y z}$ is parallel to $L(\theta)$ (for definiteness, let $\mathbf{x y z}$ be arranged counter-clockwise). There are points $\mathbf{y}_{0}$ and $\mathbf{z}_{0}$ such that a triangle of the type described maximizes the area amongst all such triangles if and only if $\mathbf{y}=\mathbf{y}_{0}$ and $\mathbf{z}=\mathbf{z}_{0}$.

Proof. Let $\mathbf{x y z}$ and $\mathbf{x}^{\prime} \mathbf{y}^{\prime} \mathbf{z}^{\prime}$ be two triangles maximizing the area (see Figure 3). First note that the area does not depend on the point lying on $L(\theta)$. Therefore, we may take $\mathbf{x}=\mathbf{x}^{\prime}$ without loss of generality. Let the altitudes of the two triangles taken from $\mathbf{x}$ be $h$ and $h^{\prime}$, and their area be $A$, so that their bases have lengths $2 A / h$ and $2 A / h^{\prime}$. By convexity, there is an inscribed triangle with base parallel to $L(\theta)$ of 
altitude $\frac{1}{2}\left(h+h^{\prime}\right)$ and base length at least $A\left(\frac{1}{h}+\frac{1}{h^{\prime}}\right)$. Since its area, $\frac{1}{4} A\left(2+\frac{h^{\prime}}{h}+\frac{h}{h^{\prime}}\right)$, must not be greater than $A$, we have that the altitudes of the two triangles, and their base length, are equal. If the two bases are not identical, then their convex hull gives the base of an inscribed triangle of the type described with greater area. Therefore, the bases must be identical.

We denote by $A(\theta)$ the maximal area of a triangle of the type described in Lemma 1, and call a triangle achieving this area a triangle anchored at $L(\theta)$ (or simply at angle $\theta$ ). Clearly, the set of all triangles anchored at support lines of $K$ contains all the critical triangles of $K$ and $\Delta(K)=2 \max _{0 \leq \theta<2 \pi} A(\theta)=2 A_{\max }$. Note that any critical triangle occurs as a triangle anchored at three angles (parallel to its three sides) and its symmetric image occurs as a triangle anchored at three more angles. We label these angles $\theta_{i}, i=1, \ldots, 6$, such that $\theta_{1}<\theta_{2}<\ldots<\theta_{6}<\theta_{1}+2 \pi$ and $\theta_{i+3}=\theta_{i}+\pi$ (the label $i$ is understood to extend cyclically such that $\left.\theta_{i+6}=\theta_{i}+2 \pi\right)$. We now show that the angles associated with two critical triangle intersperse.

Lemma 2. Let $\theta_{1}, \theta_{3}$, and $\theta_{5}$ and $\theta_{1}^{\prime}, \theta_{3}^{\prime}$, and $\theta_{5}^{\prime}$ be the angles at which two distinct critical triangles of $K$ arise, then there is some even integer $k$ such that $\theta_{1} \leq \theta_{k+1}^{\prime} \leq \theta_{3} \leq \theta_{k+3}^{\prime} \leq \theta_{5} \leq \theta_{k+5}^{\prime} \leq \theta_{1}+2 \pi$.

Proof. Let $\mathbf{x}, \mathbf{y}$, and $\mathbf{z}$ be the vertices incident on the support lines $L\left(\theta_{1}\right), L\left(\theta_{3}\right)$, and $L\left(\theta_{5}\right)$ respectively, and similarly denote by $\mathbf{x}^{\prime}, \mathbf{y}^{\prime}$, and $\mathbf{z}^{\prime}$ the vertices of the other critical triangle. If the two triangles are anchored at a common support line, then by Lemma 1 they also have two vertices in common, and the two remaining vertices lie on a line parallel to the line connecting the shared vertices. The lemma in that case then follows easily (see top panel of Figure 4). Therefore, we may assume below that all six angles are distinct. We also assume that the domain is not a parallelogram, for which the lemma can also be easily checked to hold (the details are left to the reader). Therefore, there is no line segment in the boundary of $K$ containing three of the six vertices.

We will make use of the fact that if $A B C D E$ is a non-degenerate convex pentagon, and area $(A B D) \leq \operatorname{area}(A B E)$ then area $(A C D)<$ area $(A C E)$. This fact is proved, for example, in Ref. [2] under the name of the Pentagon Lemma. A more visual argument, not using this lemma is given in Figure 4 .

First, consider the case that the triangles have a vertex in common, which without loss of generality may be taken to be $\mathbf{x}=\mathbf{x}^{\prime}$. Since the 

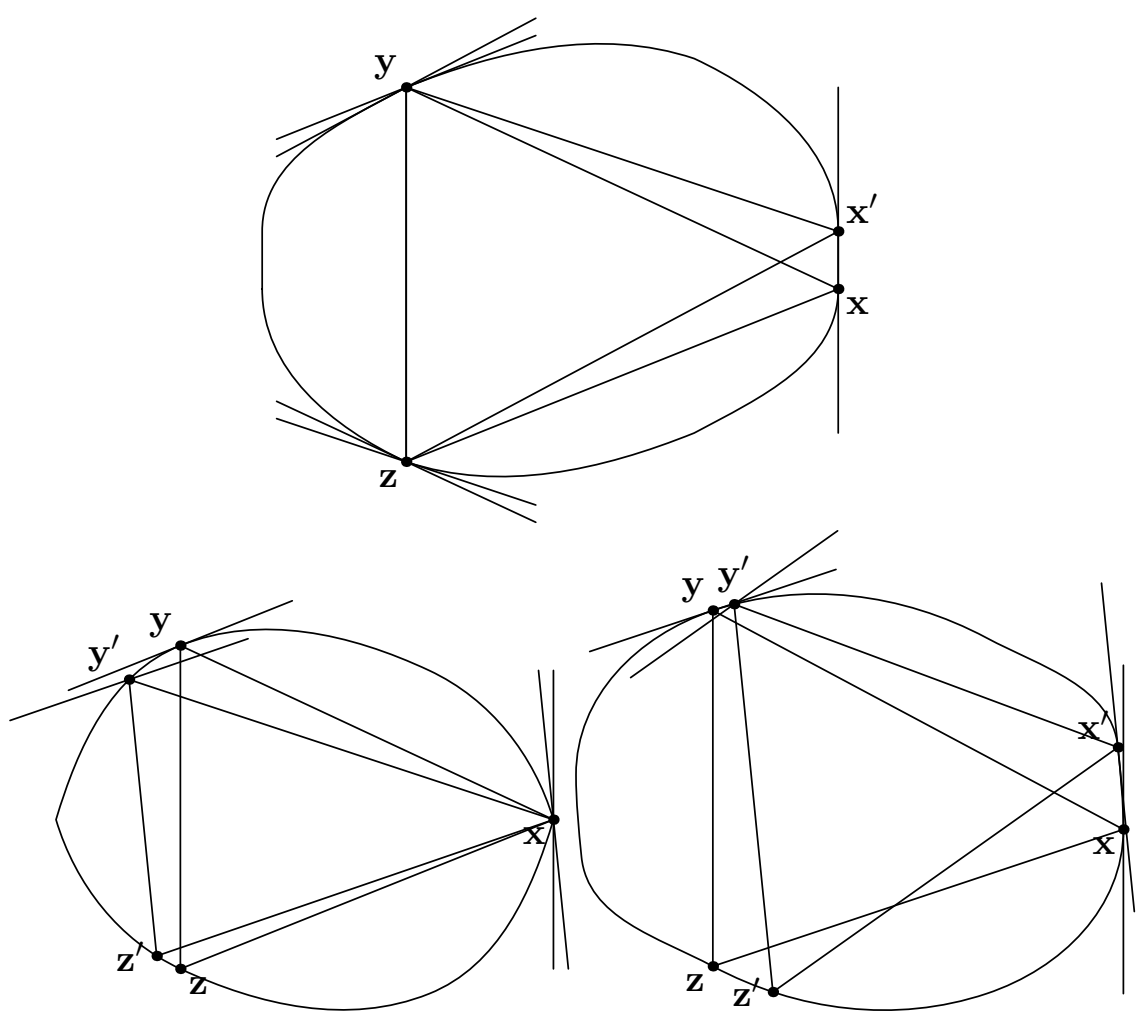

Figure 4. Three cases of the relative situation of two critical triangles of a domain, which occur in the proof of Lemma 2. In the top panel, the triangles are anchored at a common support line, and necessarily also share two vertices (see Lemma 1). As can be verified by inspection, the angle triplets at which the two triangles are anchored intersperse. The bottom-left panel illustrates the impossibility of the case that two critical triangles share a vertex and the opposite sides do not intersect: the support line through $\mathbf{y}^{\prime}$ must be parallel to $\mathbf{x z}^{\prime}$, and therefore less steep than the support line through $\mathbf{y}$, which is parallel to xz. However, from convexity of $K$, the opposite must also hold. The bottom-right panel illustrates the case that two critical triangles share no vertices or support lines, but their vertices do not intersperse along the boundary. This case is also shown to be impossible: the support line through $\mathbf{y}^{\prime}$ must be parallel to $\mathbf{x}^{\prime} \mathbf{z}^{\prime}$, and therefore steeper than the support line through $\mathbf{y}$, but again, from convexity of $K$, the opposite must also hold. 
triangles must intersect not only at a vertex (both must contain the origin), the order of the vertices as we travel counter-clockwise around the boundary cannot be $\mathbf{x y z y} \mathbf{y}^{\prime} \mathbf{z}^{\prime} \mathbf{x}$ or $\mathbf{x} \mathbf{y}^{\prime} \mathbf{z}^{\prime} \mathbf{y z x}$. If the order is $\mathbf{x y} \mathbf{y}^{\prime} \mathbf{z}^{\prime} \mathbf{z x}$, then since area $\left(\mathbf{x y z} \mathbf{z}^{\prime}\right) \leq \operatorname{area}(\mathbf{x y z})$, the Pentagon Lemma gives that $\operatorname{area}\left(\mathbf{x y}^{\prime} \mathbf{z}^{\prime}\right)<\operatorname{area}\left(\mathbf{x y}^{\prime} \mathbf{z}\right)$ : a contradiction. (see also Figure 4 for a visual illustration of why this order is impossible.) The same argument, with the triangles transposed also eliminates the order $\mathbf{x y}^{\prime} \mathbf{y z z} \mathbf{z}^{\prime} \mathbf{x}$. The order must then be $\mathbf{x} \mathbf{y} \mathbf{y}^{\prime} \mathbf{z z}{ }^{\prime} \mathbf{x}$, or equivalently $\mathbf{x y}^{\prime} \mathbf{y} \mathbf{z}^{\prime} \mathbf{z x}$. Since we know that the angle of support lines through points on the boundary cannot decrease as the point is moved counter-clockwise along the boundary, it is enough to observe that $\theta_{1}<\theta_{1}^{\prime}$ in the first case, since the corresponding support lines are parallel to $\mathbf{y z}$ and $\mathbf{y}^{\prime} \mathbf{z}^{\prime}$, and similarly that $\theta_{1}>\theta_{1}^{\prime}$ in the other case.

Now, consider the case that none of the vertices coincide. We must eliminate all cases in which one of the three $\operatorname{arcs} \mathbf{x y}, \mathbf{y z}$, or $\mathbf{z x}$ includes at least two of the vertices $\mathbf{x}^{\prime}, \mathbf{y}^{\prime}$, or $\mathbf{z}^{\prime}$. Since the triangles must intersect, it includes exactly two. Without loss of generally, we may consider the case where the order is $\mathbf{x x}^{\prime} \mathbf{y}^{\prime} \mathbf{y z z} \mathbf{z}^{\prime} \mathbf{x}$. From the fact that area $\left(\mathbf{x y}^{\prime} \mathbf{z}\right) \leq \operatorname{area}(\mathbf{x y z})$ and area $\left(\mathbf{x}^{\prime} \mathbf{y} \mathbf{z}^{\prime}\right) \leq \operatorname{area}\left(\mathbf{x}^{\prime} \mathbf{y}^{\prime} \mathbf{z}^{\prime}\right)$, the Pentagon Lemma gives that area $\left(\mathbf{x}^{\prime} \mathbf{y}^{\prime} \mathbf{z}\right)<\operatorname{area}\left(\mathbf{x}^{\prime} \mathbf{y z}\right)$ and area $\left(\mathbf{x}^{\prime} \mathbf{y z}\right)<$ area $\left(\mathbf{x}^{\prime} \mathbf{y}^{\prime} \mathbf{z}\right)$ : a contradiction. (see also Figure 4 for a visual illustration of why this order is impossible.) Consequently, the vertices of the two triangles must alternate around the boundary, and the lemma holds.

Intuitively, a domain can only be inextensible if it has a one parameter family of critical lattices. Such a situation is needed and sufficient to make sure that any augmentation of the domain would allow one of the critical lattices to increase in determinant. We prove this now.

Theorem 1. $K$ is inextensible if and only if $A(\theta)$ is constant.

Proof. First assume that $K$ is extensible. Then there exists a proper superdomain $K^{\prime} \supset K$ such that $\max A^{\prime}(\theta)=\max A(\theta)$. There exists a support line $L\left(\theta_{0}\right)$ of $K$ which intersects the interior of $K^{\prime}$. Since the triangle anchored at $L^{\prime}\left(\theta_{0}\right)$ must be of greater area than the triangle anchored at $L\left(\theta_{0}\right)$, we have $A\left(\theta_{0}\right)<A^{\prime}\left(\theta_{0}\right) \leq \max A^{\prime}(\theta)=\max A(\theta)$, and $A(\theta)$ is not constant.

For the converse assume that $A(\theta)$ is not constant. Note that $A(\theta)$ must be continuous in $\theta$, so the set of angles such that $A(\theta)<A_{\text {max }}$ is open. Consider those support lines $L(\theta)$ of $K$ that are not tangent to $K$ on either side of their intersection with the boundary, that is, support lines such that support lines for sufficiently nearby angles have 


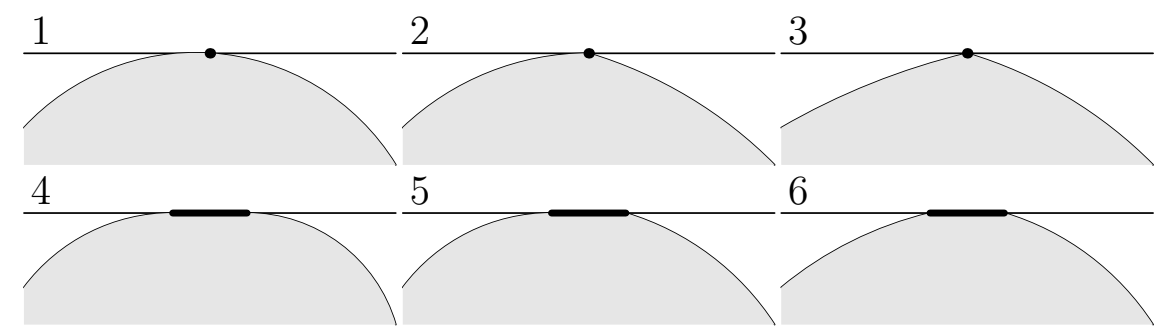

FiguRE 5. Six possible types of support lines: (1) intersecting the boundary at a point and tangent to the boundary on both sides, (2) intersecting the boundary at a point and tangent on one side, (3) intersecting the boundary at a point and tangent on neither side, (4) intersecting the boundary at a segment and tangent on both sides of the segment, (5) intersecting the boundary at a segment and tangent on one side of the segment, and (6) intersecting the boundary at a segment and tangent on neither side of the segment. For the purposes of the proof of Theorem 1, we call all the above cases tangent except for the case (3).

the same intersection with the boundary. Call support lines of this type non-tangent and call all other support lines tangent (see Figure 5).

We assume first that there is an angle $\theta$ such that $L(\theta)$ is tangent and $A(\theta)<A_{\max }$. Since sufficiently nearby angles also have suboptimal anchored triangles, we must have two support lines $L\left(\theta^{\prime}\right)$ and $L\left(\theta^{\prime \prime}\right)$, whose intersections with the boundary of $K$ are disjoint, and such that for all $\theta \in\left[\theta^{\prime}, \theta^{\prime \prime}\right]$, we have $A(\theta)<A_{\max }$. Therefore, by filling in the area between $K, L\left(\theta^{\prime}\right)$ and $L\left(\theta^{\prime \prime}\right)$, we may construct superdomains $K_{\varepsilon}^{\prime} \supset K$ with the same support lines as $K$ for angles outside $\left[\theta^{\prime}, \theta^{\prime \prime}\right]$ (and the symmetric interval $\left.\left[-\theta^{\prime},-\theta^{\prime \prime}\right]\right)$ and support lines that are moved by no more than $\varepsilon$ for the angles in $\left[\theta^{\prime}, \theta^{\prime \prime}\right]$ (see Figure [6). It is clear that $\Delta\left(K_{\varepsilon}^{\prime}\right)=\Delta(K)$ for small enough $\varepsilon$ and $K$ is extensible. Otherwise, there must be for all $\varepsilon>0$ a critical triangle of $K_{\varepsilon}^{\prime}$ of area greater than $A_{\max }$ and with a vertex on the part of the boundary of $K_{\varepsilon}^{\prime}$ that is not shared with $K$. The existence of these triangles would imply the existence of an anchored triangle for $K$ anchored at an angle $\theta \in\left[\theta^{\prime}, \theta^{\prime \prime}\right]$ and with area at least $A_{\max }$, and is therefore impossible.

On the other hand, assume that all angles for which $A(\theta)$ is submaximal have support lines that are non-tangent. Let us consider an 


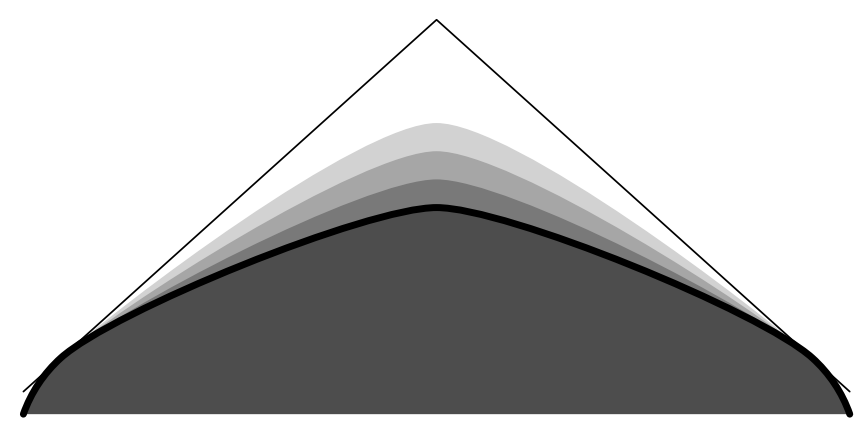

FiguRE 6. If the intersections of the support lines $L\left(\theta^{\prime}\right)$ and $L\left(\theta^{\prime \prime}\right)$ (thin lines) with the boundary of the domain $K$ (darkest gray, boundary in thick line) are disjoint, then we can construct superdomains (shades of gray) whose support lines are identical outside the intervals $\left[\theta^{\prime}, \theta^{\prime \prime}\right]$ and $\left[-\theta^{\prime},-\theta^{\prime \prime}\right]$, and arbitrarily close to the original support lines inside the intervals.

angle $\theta$ for which $A(\theta)$ is submaximal and denote as $\mathbf{x}$ the point at which $L(\theta)$ intersects $\partial K$. By continuity, $\theta$ must be part of an open interval $\left(\theta_{1}, \theta_{1}^{\prime}\right)$ such that $A\left(\theta_{1}\right)=A\left(\theta_{1}^{\prime}\right)=A_{\max }$ and $A\left(\theta^{\prime}\right)<A_{\max }$ for all $\theta^{\prime} \in\left(\theta_{1}, \theta_{1}^{\prime}\right)$. Since all angles $\theta^{\prime} \in\left(\theta_{1}, \theta_{1}^{\prime}\right)$ are submaximal, their support lines are non-tangent, and therefore both $L\left(\theta_{1}\right)$ and $L\left(\theta_{1}^{\prime}\right)$ contain $\mathbf{x} \in \partial K$, and $\mathbf{x}$ is a vertex of two critical triangles anchored at $L\left(\theta_{1}\right)$ and $L\left(\theta_{1}^{\prime}\right)$. Denote the other two angles anchoring each of these triangles as $\theta_{3}$ and $\theta_{5}$ and $\theta_{3}^{\prime}$ and $\theta_{5}^{\prime}$. Consider the intervals $\left(\theta_{3}, \theta_{3}^{\prime}\right)$ and $\left(\theta_{5}, \theta_{5}^{\prime}\right)$. By the interspersing property (Lemma 2), $A(\theta)$ is submaximal at all angles of these intervals, and therefore all angles of these intervals must have non-tangent support lines. It follows that all vertices of the two critical triangles coincide. Since this is a contradiction, the case where all submaximal angles have non-tangent support lines is impossible.

Genin and Tabachnikov have observed that convex domains, not necessarily symmetric, such that each support line anchors a critical triangle can be characterized by the equivalent property that each point on its boundary lies on an outer billiard triangle, that is, a circumscribed triangle such that the midpoint of each side is on the boundary of the domain [3]. For conciseness, we will say that a domain (again, not necessary symmetric) that possesses these equivalent properties has a circle of critical triangles. The theorem identifies the family of inextensible symmetric convex domains with the family of symmetric convex 


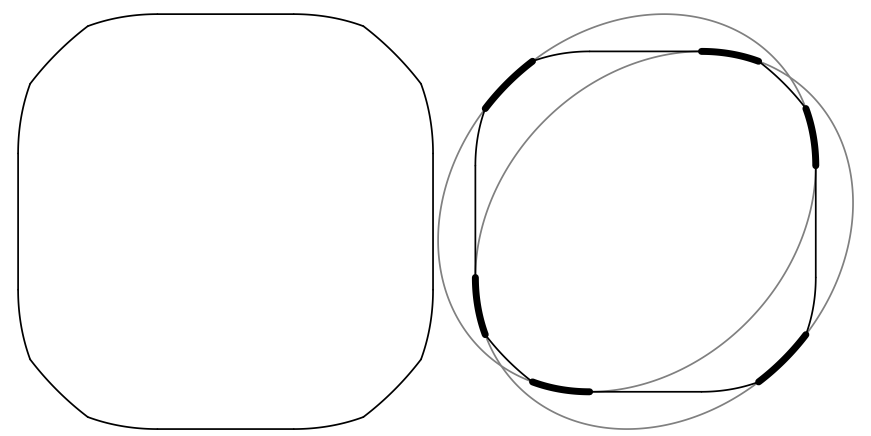

Figure 7. An example of an inextensible domain (left). The boundary of the domain is composed of 4 line segments and 12 arcs belonging to 4 ellipses. The three arcs belonging to each ellipse are traced out by the vertices of critical triangles of that ellipse (right). This construction yields a one-parameter family of inextensible domains interpolating between the disk and the square.

domains with a circle of critical triangles. Examples of inextensible domains include, in addition to the parallelogram and ellipse, the regular $6 n+4$-gons. Another example of a family of inextensible domains interpolating between the disk and the square is given in Figure 7 .

Genin and Tabachnikov conjecture that, among convex domains with a circle of critical triangles of a given area, the ellipse maximizes the area of the domain. This in fact follows easily from the following theorem of Sas:

Theorem 2. (Sas [8]) Let $K$ be a convex domain and $T_{n}$ the inscribed $n$-gon of maximal area in $K$, then

$$
\frac{\operatorname{area} T_{n}}{\text { area } K} \geq \frac{n}{2 \pi} \sin \frac{2 \pi}{n}
$$

with equality for, and only for, an ellipse.

Corollary 1. Let $K$ be a convex domain with a circle of critical triangles of area $A$, then area $K \leq 4 \pi A / \sqrt{27}$, with equality for, and only for, an ellipse.

\section{REFERENCES}

1. R. P. Bambah and C. A. Rogers, Covering the plane with convex sets, Journal of the London Mathematical Society s1-27 (1952), no. 3, 304-314.

2. D. P. Dobkin and L. Snyder, On a general method for maximizing and minimizing among certain geometric problems, 20th Annual Symposium on Foundations of Computer Science, 1979, pp. $9-17$. 
3. D. Genin and S. Tabachnikov, On configuration spaces of plane polygons, subRiemannian geometry and periodic orbits of outer billiards, J. Modern Dynamics 1 (2007), 155.

4. P. M. Gruber, Convex and discrete geometry, Springer, New York, 2007.

5. K. Mahler, On lattice points in n-dimensional star bodies $I-I V$, Proceedings of the Koninklijke Nederlandse Academie van Wetenschappen 49 (1946), 331-343, $444-454,524-532,622-631$.

6. _ On irreducible convex domains, On the area and the densest packing of convex domains, On the minimum determinant and the circumscribed hexagons of a convex domain, Proceedings of the Koninklijke Nederlandse Academie van Wetenschappen 50 (1947), 98-107, 108-118, 326-337.

7. K. Reinhardt, Über die dichteste gitterförmige Lagerung kongruente Bereiche in der Ebene und eine besondere Art konvexer Kurven, Abh. Math. Sem., Hamburg, Hansischer Universität, Hamburg 10 (1934), 216.

8. E. Sas, Über eine Extremumeigenschaft der Ellipsen, Compositio Math. 6 (1939), 468.

9. L. Fejes Tóth, Lagerungen in der Ebene, auf der Kugel und im Raum, Springer, Berlin, 1972.

Yoav Kallus, Center for Theoretical Science, Princeton UniverSity, Princeton, New Jersey 08544 